International Journal of Engineering \& Technology, $7(2.33)(2018) 165-170$
International Journal of Engineering \& Technology
SPC
Website: www.sciencepubco.com/index.php/IJET
Research paper

\title{
A study on design and implementation of dynamic location tracking system for locating remote control
}

\author{
Sung-Hyun Seo ${ }^{1}$, Kwang-Hyun Ro ${ }^{2}$ * \\ ${ }^{1}$ Doctor's course, Department of Smart Convergence Consulting, General graduate school, Hansung University, Korea \\ ${ }^{2}$ Professor, Department of Smart Convergence Consulting, General graduate school, Hansung University, Korea \\ *Corresponding author E-mail: khrho@hansung.ac.kr
}

\begin{abstract}
With the development of IoT technology, there is a growing demand for location based services for checking the mobility and identity of users, and remote controls for remote control of household appliances or electronic devices are used for home appliances or electronic devices there are many functions that are not available.

In this case, when the remote controller is lost, there are difficulties in that a large number of home appliances or electronic devices can only be started by a limited operation or can't operate various operations.

Especially, many people are losing a lot of remote control in everyday life, and the problem of wasting a considerable amount of time in finding a remote control is also happening.

In order to solve this problem, the present invention proposes a method of opening a remote control search application content, registering a unique product number assigned to the remote control, generating a location notification request signal for requesting the location information of the remote controller, And then displays the captured location or dynamic model detected from the location notification response signal on the basis of the indoor framing for $2 \mathrm{D}$ or the indoor framing for $3 \mathrm{D}$, It will be able to quickly and accurately locate the remote control that emits the beacon signal.

In other words, in this paper, the application contents for remote controller search are opened to register a unique product number assigned to the remote controller, a location notification request signal for requesting the location information of the remote controller, and a location notification response And then displays the captured spot or dynamic model detected from the signal on the beacon for $2 \mathrm{D}$ or the 3D model for 3D.

In the future, the research method to be carried out is limited to grasp the object information only by the remote controller (one single article object), but it is difficult to quickly recognize the object information transmitted from various objects having mobility used in the home or work We plan to implement and design an algorithm that optimizes the received signal strength of the BLE beacon so that there is no loss of beacon signal in a room that is subject to better system and multipath effects.
\end{abstract}

Keywords: Remote Control; Communication Terminal; Position Tracking; Beacon Signal; Position Notification Request Signal; Position Notification Response Signal.

\section{Introduction}

As IoT technology evolves, there is a growing demand for location based services to identify users' mobility and identity. Recently, miniaturization of semiconductors and development of wireless communication technology have been actively conducted on Ubiquitous Computing capable of providing users with desired information and services regardless of their location and environment. Among them, wireless location recognition technology for user convenience and stability has been applied in various fields such as navigation, home automation, parking management, logistics management, guide to exhibition hall, and prevention of lost children [1].

Remote control that can remotely control household appliances or electronic devices is equipped with many functions that are not available in household appliances or electronic appliances, so that when a remote control is lost, many household appliances or electronic appliances are started only in a restricted operation, It is difficult to drive the motor. As a result, the user could not enjoy the benefits of the electronic appliances or electronic devices he purchased because of the loss of the remote control, and he was forced to feel a great inconvenience.

It is difficult to keep the remote control in place because many people use the remote control according to the purpose of use. Especially, when the children are playing with it, it can be under the sofa and under the sofa backrest. This is because when you move to a room or kitchen to do something, you leave it there. As a result, there was a problem that many people spend a lot of time searching without a remote control.

In order to solve this problem, this research paper opens the app contents for searching for remote control, registers a unique product number given to the remote controller, generates a location notification request signal for requesting the location information of the remote controller, The location notification response signal is converted into a beacon signal, and then the captured location or dynamic model detected from the location notification response signal is displayed on the $2 \mathrm{D}$ modeling figure or the $3 \mathrm{D}$ modeling map for 3D, We want to be able to quickly and accurately locate the remote control that emits a beacon signal.

This research paper shows a dynamic model which is shaped as a 2D image, a 3D image, or a label attaching image in an indoor 
molding figure for $2 \mathrm{D}$ or an indoor molding figure for 3D mounted in an app for searching remote control, Even if you have a remote control, you can easily find the remote you need.

In addition, the system proposed in this paper can collectively store various remote controllers provided for various home appliances and household appliances in the app contents for finding remote control, so that users can easily see the direction of a lost or lost remote controller by user carelessness or forgetting can do. The system is able to overcome the fear of finding a remote controller for various household appliances and home appliances, so that the remote controller can be re-purchased online by linking the app contents for finding remote control with the shopping mall Thereby maximizing the user's convenience.

In addition, this research paper solves the fundamental countermeasures that do not appropriately use various functions provided by various home appliances and home appliances due to the loss of the remote controller by further spreading the application contents for finding remote control to the public, Prevent new purchases of home appliances and new appliances.

\section{Indoor and outdoor positioning technology trend}

\subsection{Overview of indoor and outdoor positioning system}

GPS (Global Positioning System) is widely used in field I that requires location tracking service because it can locate the moving object in real time. However, it is less effective in indoor rooms where reception of satellite signals is difficult. Researches on wireless communication systems such as UWB, RFID, and Zigbee have been actively pursued for location recognition technology that can be utilized in indoor environments [2-4].

The integration of ICT and manufacturing into the manufacturing sector and the creation of high value added services have become new competitiveness by increasing the efficiency of the manufacturing industry and increasing the weight of the manufacturing industries in each country. The revolution of the production method and the breakthrough of the manufacturing crisis But it is emerging as a factor to give wings to the revival of manufacturing. IC-based intelligence is applied to all fields such as smart phone, smart car, smart home, and smart city, and factory intelligence is also indispensable [5].

Research on the current position recognition and tracking can be classified into a method using a triangulation technique, a scene analysis technique for image recognition, and a position recognition using a proximity method.

In this paper, focusing on the in-door environment, we will discuss the location of objects in the indoor or shaded areas of buildings, especially the location tracking technology and system for locating, recognizing, and tracking remote controls that are frequently used at home. Let's look at [6-7].

Accordingly, the dynamic location tracking system for searching for a remote control of the present invention generates a location notification request signal for requesting location information of a remote controller identified by a unique product number assigned to the remote controller, and transmits a beacon signal, And the location information of the remote controller recorded in the beacon signal is captured as a capture point to display on the indoor 2D modeling figure or the 3D modeling figure embodied in the app contents for finding remote control, And it is a system that expresses on the indoor molding figure for $2 \mathrm{D}$ or the indoor molding figure for $3 \mathrm{D}$.

\subsection{Research trend of IoT-based positioning technology}

The research trend of IoT (Internet of Things) based location tracking technology was confirmed through previous studies.

Technology that provides various services based on location information like Internet of things, Smart Home is emerging as core technology. In order to effectively provide such services, it is im- portant to grasp the exact location of the user in the indoor environment. Accordingly, various researches have been actively conducted to improve the accuracy of the indoor location recognition technology. In particular, indoor location estimation technology using a BLE (Bluetooth Low Energy) beacon is attracting attention. BLE beacons have a great advantage of low power consumption and low cost because they have low battery consumption, and have a long serviceable range of about $50 \mathrm{~m}$. In addition, frequency hopping techniques can be used to avoid collisions with other devices.

Beacon was originally used to refer to devices that transmit signals periodically to convey information and other information, such as beacons and lighthouses. The beacon serves as a reference point for locating, and communication techniques such as ultrasound, infrared, and Bluetooth are required to actually transmit information. Depending on the type of signal transmission, beacons using ultrasound or visible light, sound-based low-frequency beacons, LED beacons, Wi-Fi beacons, and Bluetooth beacons can be categorized. In recent years, a BLE-based short-range wireless communication device is generally called a beacon. To use the beacon service in your application, you need a smartphone with Android 4.3 or higher, iOS 7.0 or higher.

One of the advantages of BLE beacons is that it can be used for a long period of about 2 years with one small coin battery because it consumes less battery than existing technology. Second, the frequency hopping technique is used to continuously transmit and change frequencies in order to avoid collisions with other devices that use the ISM frequency band that can be used without separate permission. Third, one of the RFID technologies has a merit that it can communicate up to about $50 \mathrm{~m}$ by using a longer distance than NFC(Near Filed Communication) using the frequency band of $13.56 \mathrm{MHz}$. As such, BLE beacons are attracting attention as indoor location services using beacons because of their low power and low cost features and applicability to various applications [8].

\section{Design of dynamic location tracking system for finding remote control}

\subsection{Overview of dynamic location tracking system for locating remote controls}

In this paper, a dynamic location tracking system for finding a remote controller registers a unique product number assigned to a remote controller using application contents for finding a remote controller, converts a location notification response signal responding to the location notification request signal into a beacon signal, And to design and propose a system for displaying the captured spot or dynamic model detected from the detected point or $2 \mathrm{D}$ on an indoor framing for $2 \mathrm{D}$ or an indoor framing for $3 \mathrm{D}$.

\subsection{Construction and operation of dynamic location tracking system for locating remote control}

As shown in figure 1 and figure 3, the dynamic location tracking system for finding a remote controller is largely composed of a communication terminal, a remote controller, and a management server, and a more detailed system configuration and operation description are implemented as described below.

That is, the communication terminal opens the remote-control search application content, stores the unique product number assigned to the remote controller, matches the unique product number called on the matching table with the check box marked with the usage name 1: 1, And generates a position notification request signal for requesting the position information of the remote controller when selecting the application name (ex: TV button, air conditioner button, etc.) displayed on the remote controller registration list. 


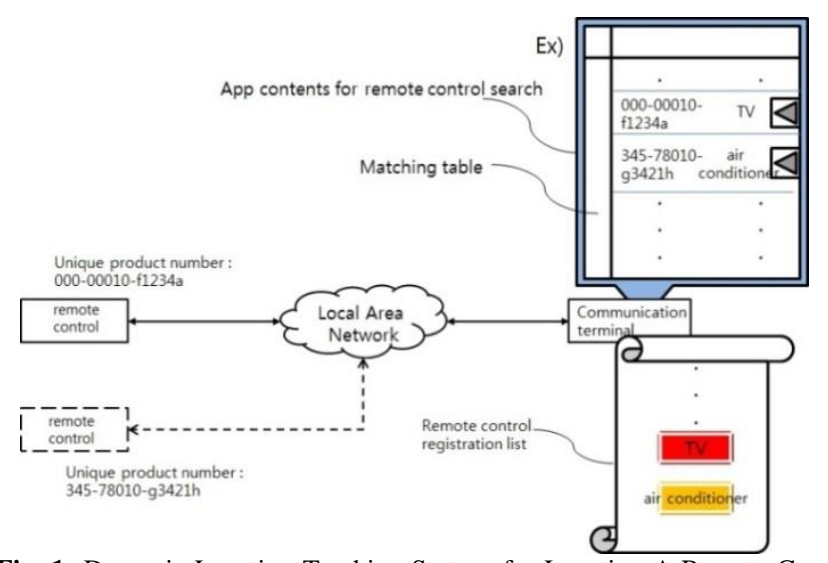

Fig. 1: Dynamic Location Tracking System for Locating A Remote Controller and Operation of Communication Terminal Associated Therewith.

As the beacon signal is transmitted to the communication terminal via the local area network, the communication terminal analyzes the location information of the remote controller recorded in the beacon signal as shown in Fig. 2, detects the acquisition point, Or an indoor molding figure for $3 \mathrm{D}$, or the acquisition point is expressed as a dynamic model of a 2D image, a 3D image, or a label attaching image, and is expressed on an indoor molding figure for $2 \mathrm{D}$ or an indoor molding figure for $3 \mathrm{D}$.

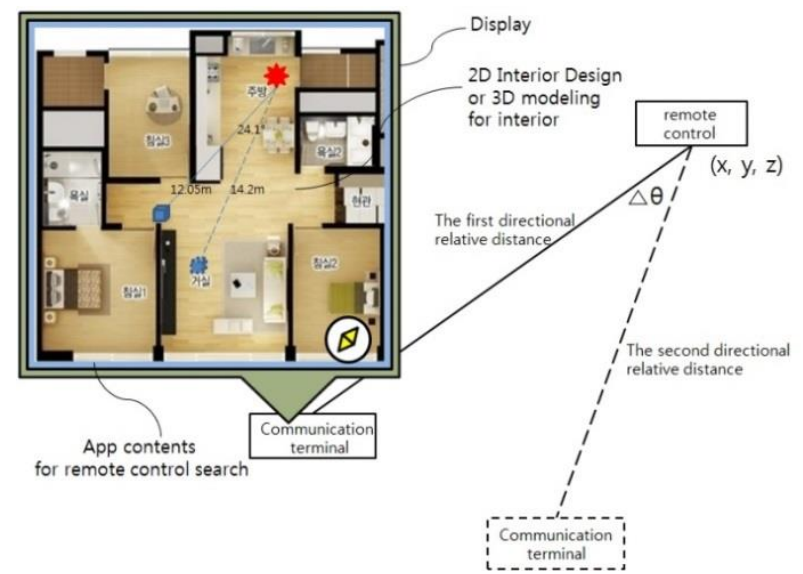

Fig. 2: Implementation of Operation of Communication Terminal to Find Remote Controller.

The detailed configuration of the communication terminal is as described below. That is, as shown in FIG. 3, the communication terminal receives a beacon signal and obtains the current position of the remote controller by using coordinate values determined from the position information of the remote controller. A central processing unit for controlling the display of the modeling figure or the indoor molding figure for $3 \mathrm{D}$, and the display unit for displaying the $2 \mathrm{D}$ molding model or the $3 \mathrm{D}$ molding model into which the acquisition point or the dynamic model is inserted.

The position tracking unit calculates the distance by which the first and second directional relative distances to the remote controller are calculated by using the beacon signals transmitted from the first antenna and the second antenna respectively and the first and second calculated sensitivity of the communication terminal And a direction calculating section for calculating a dispatching direction $(\Delta \theta)$ indicating the acquisition point where the beacon signal is transmitted based on the first, second, and first and second reception sensitivities, that is, $\Delta \theta=\theta^{1}-\theta^{2}$ ([Equation 5]).

When the current position of the remote controller is searched for on the remote control search application content, the display unit displays a dispatch direction $(\Delta \theta)$ Indicating the acquired capture point (i.e., the point where the beacon signal is transmitted) under the control of the central processing unit Arrows are displayed or displayed in units of one of 360 degrees. The display shows the first and second directional relative distance from the remote con- troller in meters $(\mathrm{m})$ or centimeters $(\mathrm{cm})$ by reference to the coordinate values [see Figure 2].

In general, the receiver sensitivity is a value obtained by adding the noise figure because the signal-to-noise ratio is degraded at the output stage because the noise included in the receiver is included by the noise figure as compared with the antenna input.

The thermal noise density is a value that should be basically obtained when calculating all the reception sensitivities.

Since the thermal noise density appears at all frequencies, the thermal noise amount becomes the same if the reception frequency bandwidth is constant even if the frequency band is different.

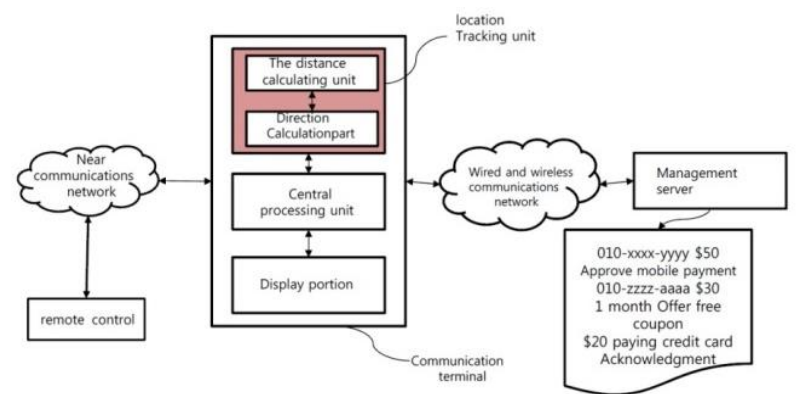

Fig. 3: The Details of the Communication Terminal Searching for the Remote Controller and the Overall System.

That is, the communication terminal first obtains the first and second thermal noise densities $\left(\mathrm{N}_{0}^{1}, \mathrm{~N}_{0}^{2}\right)$ generated in the communication terminal to find the first and second dispatching directions $\left(\theta^{1}, \theta^{2}\right)$ for the first and second acquisition points, 3 ) ([Equation 1]), and the first and second reception sensitivities (RSSI 1 and $\mathrm{RSSI}_{2}$ ) are obtained based on the first and second thermal noise densities $\left(\mathrm{N}_{0}^{1}, \mathrm{~N}_{0}^{2}\right)$.

Hereinafter, the above-mentioned matters are summarized on the basis of the following equations. The first and second thermal noise densities generated in the communication terminal can be obtained through the following [Equation 1]. To this end, four plate antennas mounted in the communication terminal receive a beacon signal from the remote controller.

Also, the communication terminal calculates first and second transmission losses $\mathrm{NF}_{1}$ and $\mathrm{NF}_{2}$ using first and second signal-tonoise ratios $\left(\mathrm{SNR}_{0}^{1}, \mathrm{SNR}_{0}^{2}\right)$ of the output stage mounted in the cabin, and then calculates the first and second thermal noise densities, The first and second reception sensitivities are obtained by substituting the transmission loss $\mathrm{NF}_{1}$ and $\mathrm{NF}_{2}$ and the first and second reception sensitivities $\mathrm{RSSI}_{1}$ and $\mathrm{RSSI}_{2}$ and the reception bandwidth into the reception sensitivity calculation [Equation 2].

\section{【Equation1 】}

$\mathrm{N}_{0}^{1}=10 \log \left(\mathrm{KT}_{1}\right)[\mathrm{dBm} / \mathrm{Hz}]$

$\mathrm{N}_{0}^{2}=10 \log \left(\mathrm{KT}_{2}\right)[\mathrm{dBm} / \mathrm{Hz}]$

Here, $\mathrm{N}_{0}^{1}$ : the first thermal noise density, $\mathrm{N}_{0}^{2}$ : the second thermal noise density, $\mathrm{K}$ : Boltzmann constant, $\mathrm{T}_{1}$ : first absolute temperature, $\mathrm{T}_{2}$ : first absolute temperature

\section{【Equation2】}

First reception sensitivity

$\left(R S S I_{1}\right)=N_{0}^{1}+10 \log B+N F_{1}[\mathrm{dBm}]$,

Second reception sensitivity

$\left(R S S I_{2}\right)=N_{0}^{2}+10 \log B+N F_{2}[\mathrm{dBm}]$

$N F_{1}=10 \log \frac{S N R_{i}}{\left(S N R_{o}^{1}\right)}[\mathrm{dBm}]$ 
$N F_{2}=10 \log \frac{S N R_{i}}{\left(S N R_{o}^{2}\right)}[\mathrm{dBm}]$

$S N R_{0}^{1}=\frac{S_{o}^{1}}{N_{o}^{1}}=\frac{A S_{i}}{\left(A N_{i}+N_{\text {inside }}^{1}\right)}$

$S N R_{0}^{2}=\frac{S_{o}^{2}}{N_{o}^{2}}=\frac{A S_{i}}{\left(A N_{i}+N_{\text {inside }}^{2}\right)}$

$A=20 \log \frac{V_{o}}{V_{i}}$

Here, $\mathrm{NF}_{1}$ is a first noise figure, $\mathrm{NF}_{2}$ is a second noise figure, $\mathrm{B}$ is a receiving bandwidth, $S N R_{0}^{1}$ is the first signal-to-noise ratio of the output terminal, $S N R_{0}^{2}$ is the second signal-to-noise ratio of the output terminal, $\mathrm{A}$ is the amplification degree, $\mathrm{V}_{\mathrm{o}}$ is the output voltage of the communication terminal, $\mathrm{V}_{\mathrm{i}}$ is the input voltage

Also, the communication terminal receives the first and second position notification response signals(That is, the first and second beacon signals), which are response signals for the first and second position notification request signals transmitted to grasp the position of the remote controller, through the four plate-shaped antennas.

The communication terminal can obtain the first and second transmission losses from [Equation 3], and can calculate the first and second directional relative distances between the communication terminal and the remote controller by using the formula of Friis.

Therefore, the first directional relative distance $\mathrm{d} 1$ generated between the communication terminals moved in the first direction on the basis of the remote controller, as well as the first directional relative distance $\mathrm{d} 1$ generated between the communication terminals moved in the second direction on the basis of the remote controller after the lapse of $t$ The biaxial relative distance $\mathrm{d} 2$ can also be obtained by the following [Equation 4].

\section{【Equation3】}

First beacon signal $(\mathrm{Bcon} 1)-\mathrm{RSSI}_{1}=\mathrm{L}_{1}[\mathrm{dBm}]$

Second beacon signal $(\mathrm{Bcon} 2)-\mathrm{RSSI}_{2}=\mathrm{L}_{2}[\mathrm{dBm}]$

Here, L1: first transmission loss, L2: second transmission loss The first and second beacon signals may be referred to as first and second position notification response signals.

\section{【Equation4】}

$d 1=\frac{C}{4 \pi f} 10^{\frac{L_{1}}{20}}$

$d 2=\frac{C}{4 \pi f} 10^{\frac{L_{2}}{20}}$

Here, C: propagation velocity, f: first and second beacon signal frequency bands, d1: first directional relative distance, d2: second directional relative distance

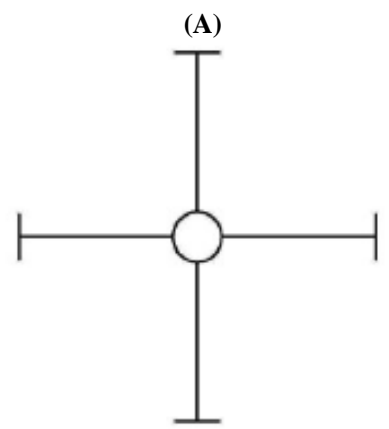

(B)

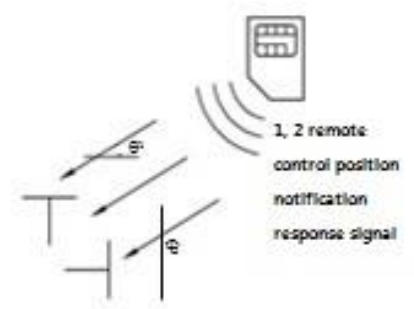

Fig. 4: (A) Four Plate Antennas Provided in the Communication Terminal, (B) the First and Second Direction Directions Measured from the First and Second Remote Controller Position Notification Response Signals Received by the First and Second Directional Antennas.

\section{【Equation5】}

$\mathrm{v}_{1}^{1}=\mathrm{KS} \sin \theta_{1}^{1}$

$\mathrm{v}_{2}^{1}=\mathrm{KS} \sin \theta_{2}^{1}$

$\mathrm{v}_{1}^{2}=\mathrm{KS} \sin \theta_{1}^{2}$

$\mathrm{v}_{2}^{2}=\mathrm{KS} \sin \theta_{2}^{2}$

Here, $v_{1}^{1}, v_{2}^{1}$ : The intensity of the first, 1'-position notification response signal received by the first and second directional antennas having the first direction in the planar antenna of the communication terminal

$\mathrm{v}_{1}^{2}, \mathrm{v}_{2}^{2}$ : The intensity of the second, 2'-position notification response signal received by the 1 ', 2 '-directional antenna having the second directionality in the planar antenna of the communication terminal

$\mathrm{k}$ : constant, S: total area of four plate antenna

$\theta^{1}=\tan ^{-1} \frac{\mathrm{v}_{1}^{1}}{\mathrm{v}_{2}^{1}}$

$\theta^{2}=\tan ^{-1} \frac{v_{1}^{2}}{v_{2}^{2}}$

$\Delta \theta=\theta^{1}-\theta^{2}$

Here, $\theta^{1}, \theta^{2}$ : The first and second dispatching directions, which indicate the acquisition points from which the first and second position notification response signals are transmitted

$\Delta \theta$ : The directional relative angle changed during the time $\mathrm{t}$ when the communication terminal moves from the first point to the second point

Finally, the communication terminal can obtain the first and second dispatch directions (d1, d2) for the first and second acquisition points where the remote controller is searched using the first and second directional relative distances $\mathrm{d} 1$ and $\mathrm{d} 2$ [9].

A first dispatching direction $\theta^{1}$ indicating a first acquisition point where the first position notification response signal is transmitted and a second dispatching direction $\theta^{2}$ indicating a second acquisition point where a second position notification response signal is transmitted is expressed by [Equation 5]. <see Figure 4>

Also, when the communication terminal moves from the first point to the second point for thours based on the remote controller, the changed directional relative angle $\Delta \theta$ between the remote controller and the communication terminal may also be obtained. In other words, if the first and second directional relative distances $\mathrm{d} 1$ and $\mathrm{d} 2$ and the relative direction are known, (Coordinates (x, y, $\mathrm{z})$ ) of the remote controller even if the communication terminal moves from the first point to the second point for $t$ hours based on the remote controller

Upon receipt of the location notification request signal transmitted through the local area network, the remote controller generates a location notification response signal responding to the location notification request signal, changes the location notification re- 
sponse signal to a beacon signal, It periodically repeats transmission, and at the same time, illuminates the LED indicator of the instrument.

The detailed configuration of the remote controller is as described below. And a signal output unit for outputting a beacon signal to the outside in accordance with a signal process of the control unit. In addition, as the communication terminal delivers the placement image of the current location and the home appliance to the management server connected to the wired and / or wireless communication network, the management server displays the interior structure figure of the current address and the indoor interior molding figure reflecting the arrangement image of the home appliance, And determines whether or not to approve the online payment with the communication terminal, and transmits it to the remote control finder application contents free of charge or free of charge. Since the communication terminal already knows the first and second directional relative distances $(\mathrm{d} 1, \mathrm{~d} 2)$ and the first and second directional relative distances ( $\mathrm{d} 1$ and $\mathrm{d} 2$ ) and $\Delta \theta$ when moving from the first point to the second point for a time $t$ on the basis of the remote control, the triangular measurement method and the Pythagorean formula The distance $\mathrm{d} 3$ from the first point to the second point can be easily obtained as shown in [Equation 6]. <see Figure 2>

\section{【Equation6】}

$\mathrm{d} 3=\sqrt{(\mathrm{d} 1 \cdot \sin \Delta \theta)^{2}+(\mathrm{d} 2-(\mathrm{d} 1 \cdot \cos \Delta \theta))^{2}}$

\subsection{Operational test and implementation design of dy- namic location tracking system for finding remote con- trol}

The communication terminal opens the remote control search application content to store the unique product number assigned to the remote controller, registers the unique product number called on the matching table and the check box marked with the usage name 1: 1, and registers the remote controller

The communication terminal generates a position notification request signal for requesting the position information of the remote controller when selecting the application name displayed on the remote controller registration list.

Upon receipt of the position notification request signal through the local area network, the remote controller generates a position notification response signal responsive to the position notification request signal, and then converts the position notification response signal into a beacon signal and repeatedly transmits the beacon signal to the communication terminal periodically Turn on the LED display.
Upon receipt of the beacon signal transmitted from the local area network, the communication terminal analyzes the location information of the remote controller recorded in the beacon signal and detects the acquisition point. Thereafter, the communication terminal displays the indoor molding figure for $2 \mathrm{D}$ or the indoor molding figure for 3D mounted in the remote control search application contents.

More specifically, the communication terminal includes a position tracking unit, a central processing unit, and a display unit, and the position tracking unit includes a distance calculating unit and a direction calculating unit.

The positioning unit in the communication terminal obtains the current position of the remote controller by using the coordinate value determined from the position information of the remote controller. The central processing unit shapes the current position of the remote controller by the acquisition point or dynamic mod$\mathrm{el}$, So that it is displayed on the interior decoration figure for use. Under the control of the central processing unit, the display unit displays the dispensing direction $\Delta \theta$ indicating the first and second acquisition points at which the beacon signal is transmitted, by an arrow or in units of one of 360 degrees. The display unit displays the first and second directional relative distances (d1, d2) in units of meters $(\mathrm{m})$ or centimeters $(\mathrm{cm})$, respectively, based on the coordinate values.

To this end, the position-tracking intra-apparatus distance calculator calculates the distance between the remote controller and the remote controller using the first and second beacon signals and the first and second reception sensitivities $\mathrm{RSSI}_{1}$ and $\mathrm{RSSI}_{2}$ received from at least one plate- 1, 2 directional relative distances (d1, d2).

It is assumed that the direction calculating unit must calculate the dispatch direction $\Delta \theta$, i.e.

$\Delta \theta=\theta^{1}-\theta^{2}$, indicating the first and second acquisition points where the first and second beacon signals are transmitted based on the first and second reception sensitivities ( $\mathrm{RSSI}_{1}$ and $\mathrm{RSSI}_{2}$ ).

Accordingly, as a result of the test implemented on a real system, the communication terminal shapes the acquisition point into a $2 \mathrm{D}$ image, a 3D image, or a dynamic model of a label-attached image, and expresses it on an indoor framing diagram for $2 \mathrm{D}$ or an indoor framing for 3D. [see Figure 5]

As the communication terminal delivers the layout image of the current location and the home appliance to the management server connected to the wired / wireless communication network, the management server creates the indoor $2 \mathrm{D}$ figure or the $3 \mathrm{D}$ indoor figure drawing reflecting the internal structure of the current address and the image do.

The management server decides whether or not to approve the online settlement with the communication terminal, and then transmits the $2 \mathrm{D}$ or $3 \mathrm{D}$ framing to the remote control finder application content for free or for free.

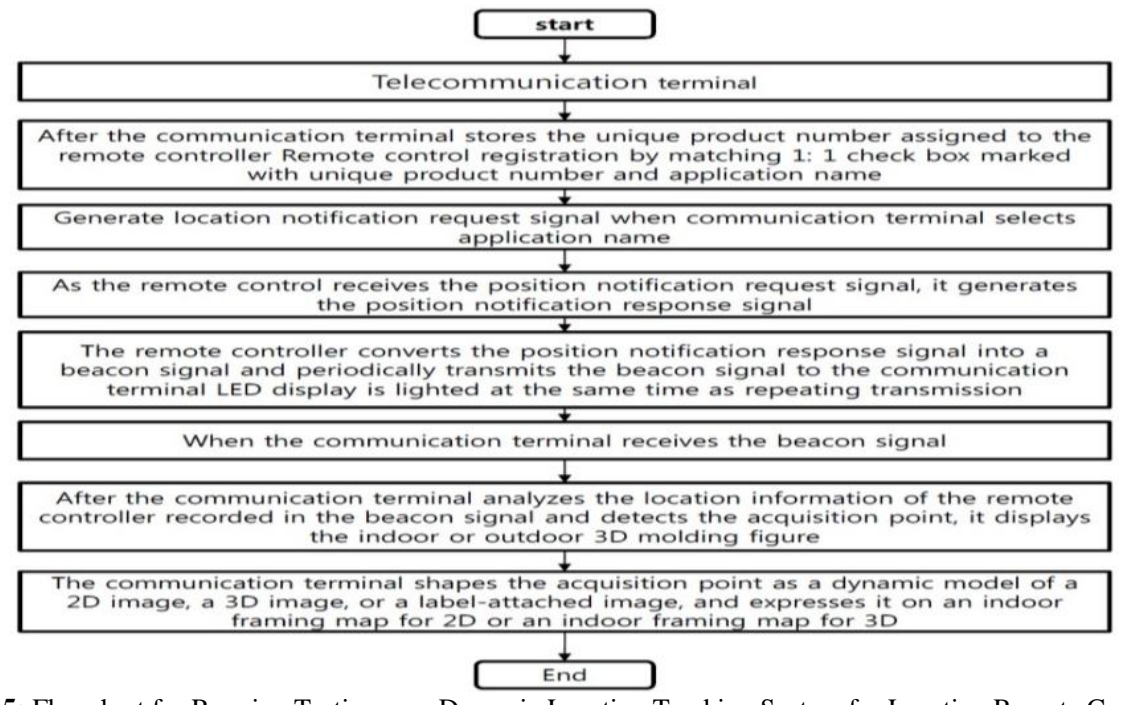

Fig. 5: Flowchart for Running Testing on a Dynamic Location Tracking System for Locating Remote Control. 


\section{Conclusion and future directions}

In this paper, a unique product number assigned to a remote controller is registered by using application contents for finding a remote control, and a location notification response signal responding to a location notification request signal is converted into a beacon signal. Then, we have designed and proposed a system that displays a model on an indoor modeling figure for $2 \mathrm{D}$ or an indoor modeling figure for 3D.

In order to make it easier to find the remote control, this study linked the communication terminal receiving the beacon signal The communication terminal confirms the object information (capture point or dynamic model) identified from the beacon signal of the remote control provided from the management server It will be available through the app contents for finding the remote molding for $2 \mathrm{D}$ indoor molding figure or $3 \mathrm{D}$ molding indoor molding figure.

In this paper, we limit the ability to grasp object information only by remote controller (single object), but in the future, we can quickly recognize object information from various objects with mobility used in home or work I plan to implement and design a better system.

In addition, it will be necessary to implement an information security algorithm according to the use of the interface of the remote control finder application realized in the communication terminal and to construct a user customized type solution so that a more variety of object information can be accessed quickly and easily.

It is necessary to study the algorithm that optimizes the received signal strength of the BLE beacon so that the loss of the beacon signal does not occur in a room affected by multipath.

We will continue to provide more reliable location-based services by combining non-contact location-awareness research based on wireless environment such as WLan, Bluetooth, RFID, and IrDA on this system developed in future contact method.

\section{References}

[1] H. J. Cho, K, I, Hwang, D, S, Rho, D, H, Seo, RealTime Indoor Positioning System using IEEE 802.15.4a and Sensors, Journal of the Korean Society of Marine Engineering, vol. 6, no. 36, pp. 850-856, Sep. 2012.

[2] B.K. Kim, W.V. Park, Y.W. Ko, Indoor positioning system using inertial sensor and cricket, The journal of Korean Institute of Information Technology, vol. 9, no.5, pp. 17-24, 2011.

[3] S. K. Park and Y. S. Suh, Pedestrian navigation system using inertial sensors and vision, Trans. KIEE, vol. 59, no. 11, 2010.

[4] B. Alzvi, and K. Pahlavan, Modeling of the TOA-based Distance Measurement Error using UWB Indoor Radio Measurement, IEEE Communication Letters, vol. 10, no. 4, pp. 275-277, 2006.

[5] Am-suk Oh, Smart Factory Logistics Management System Using House Interior Position Tracking Technology Based on Bluetooth Beacon, Journal of the Korea Institute of Information and Communication Engineering, Vol. 19, No. 11 : 2677 2682, Nov. 2015.

[6] Park Jonghyun, Kim Moon-gu, BaekJonghyun, Analysis of Industry Structure of Location-Based Services and Strategic Direction of Market Development, Journal of the Korean Institute of Communication Sciences, Volume 20, Issue 4. 2003.4.

[7] Park, Ock-Ryeong, and Sung-Hee Kim, Research Trends of Location Recognition System for Ubiquitous Computing, ETRI Weekly Technology Trend 1098, 2003.6.

[8] Park, JH, A Study on Indoor Location Recognition Technology Using BLE Beacon, Kwangwoon University, Master Degree, 2016.

[9] Korea Electronics and Telecommunications Research Institute, Apparatus and method for searching for objects, patent registration 10 1182726, patent office, 2012, 9 . 